\title{
El apoyo a la autonomía generado por entrenadores, compañeros y padres y su efecto sobre la motivación autodeterminada de deportistas de iniciación
}

\author{
Yago Ramis*, Miquel Torregrosa, Carme Viladrich, y Jaume Cruz \\ Universitat Autònoma de Barcelona, España
}

\begin{abstract}
Resumen: El objetivo de este estudio fue conocer la influencia relativa de los agentes de socialización en el deporte de iniciación sobre la motivación autodeterminada de los jóvenes deportistas. Utilizamos una metodología transversal y evaluamos, mediante cuestionarios, la capacidad predictiva de la percepción de apoyo a la autonomía generado por entrenadores, compañeros de equipo y padres sobre la regulación autónoma, la regulación controlada y la amotivación de 269 deportistas de entre 11 y 17 años, mediante análisis de regresión. Los resultados señalaron que la percepción de apoyo a la autonomía generada por los entrenadores en primer lugar, y en menor medida la de los padres y los compañeros, predecía la regulación autónoma de los deportistas. La percepción de apoyo a la autonomía generado por los entrenadores, a su vez, actuaba como factor protector de la amotivación de sus jugadores. La discusión, en la línea del modelo jerárquico de Vallerand, apunta a que en un contexto específico como es el ámbito deportivo, el apoyo a la autonomía de mayor influencia es el de la figura de más autoridad en dicho ámbito, en este caso el entrenador.

Palabras clave: Teoría de la autodeterminación, deporte, entorno social.
\end{abstract}

\section{Introducción}

Uno de los aspectos clave que se plantea desde la perspectiva de la Teoría de la Autodeterminación (SDT, Self Determination Theory; Deci y Ryan, 1985; 2000), es el papel activo de las personas en la construcción de su crecimiento personal, integridad y bienestar a partir de la satisfacción de las necesidades básicas (i.e., autonomía, competencia y relaciones). No obstante la teoría enfatiza también que el entorno y las personas que lo conforman son un elemento fundamental en la facilitación, o por el contrario la frustración de estas necesidades. Uno de los conceptos ambientales centrales para la satisfacción de las necesidades básicas es el llamado apoyo a la autonomía (Deci y Ryan, 1987), es decir, la disposición de un individuo en posición de autoridad (e.g., el entrenador) para ponerse en la posición del otro (e.g., el deportista), facilitarle información apropiada y significativa, y ofrecerle oportunidades de decisión minimizando simultáneamente la presión externa ejercida sobre él (Black y Deci, 2000). Esta facilitación del entorno no solo tendría efecto sobre la satisfacción de la necesidad básica de autonomía, sino que, promocionaría también las necesidades de competencia (e.g., Vallerand, Fortier, y Guay, 1997) y relaciones (e.g., Assor, Roth, y Deci, 2004), para finalmente favorecer formas más autodeterminadas de motivación para el individuo (Deci y Ryan, 2000).

La influencia de los otros significativos sobre las necesidades básicas y la motivación de los individuos se ha concretado a nivel teórico en el Modelo Jerárquico de la Motivación de Vallerand (2007). Este autor propone que la relación de los factores sociales sobre los aspectos individuales dentro de la autodeterminación, se puede producir en tres niveles de ge-

* Dirección para correspondencia [Correspondence address] Yago Ramis, E-mail: Yago.Ramis@uab.cat
Title: Coaches, peers and parents' autonomy support and its predictive capacity on young athletes' self-determined motivation.

Abstract: The purpose of this study was to explore the influence of youth sports' socialization agents on athletes' self-determined motivation. We used a transversal methodology and assessed, via questionnaires, the predictive capacity of coaches, peers and parental autonomy support on autonomous regulation, controlled regulation and amotivation of 269 athletes age ranged between 11 and 17 years old, through regression analyses. Results showed that the perception of coaches' autonomy support in first place, followed by parental and peers', predicted a more autonomous behavioral regulation. The perception of coaches' autonomy support also played the role of protective factor for the athletes' amotivation. Our discussion, in line with Vallerand's hierarchical model, suggests that in a specific context such as sports, the most influent autonomy support would be provided by the most authoritative figure in that given context, specifically coaches for this case.

Key Words: Self determination theory, sports, social environment.

neralidad: (a) el Nivel Situacional se refiere a la motivación experimentada por el individuo en el momento en que está participando en una actividad concreta; (b) el Nivel Contextual tiene relación con la motivación habitual que un individuo siente respecto de un contexto específico (e.g., académico, deportivo, interpersonal); y (c) el Nivel Global que se refiere a la orientación motivacional general del individuo de cara a interactuar con el entorno que le rodea. Este modelo, entre sus postulados fundamentales plantea que los aspectos ambientales a un nivel específico, afectarán a las variables individuales de su mismo nivel; que en cuanto al nivel de generalidad, los contextos más concretos varían o se pueden modificar con mayor facilidad que los niveles más generales; y que los tres niveles se conectan bidireccionalmente de forma ascendente y descendente, por lo que los cambios a nivel general afectarán a la motivación en contextos y situaciones específicas y viceversa, los cambios de motivación para un contexto o situación, pueden modificar la orientación motivacional global del individuo. En este sentido, se extrae que los diferentes agentes sociales podrán influir en diferentes niveles de generalidad, así los padres afectarían a un nivel global (Assor et al., 2004; White, 1996) puesto que son una referencia a lo largo de los diferentes contextos de la vida de los jóvenes; en el contexto específico del deporte, los entrenadores representarían la figura jerárquica de referencia (Hagger y Chatzisarantis, 2005; Wylleman y Lavallee, 2004) determinando los valores y metas en el seno del equipo; finalmente, el grupo de iguales, si bien su papel no se ha estudiado con la misma profundidad que el de padres y entrenadores, marcarían el grado de aceptación y pertenencia al grupo, así como la consolidación de uno u otro clima motivacional (Brustad, 1996; Ntoumanis y Vazou, 2005; Smith, 2003). 
Desde la conceptualización original de Deci (1975), se presume que las personas pueden presentar dos tipos fundamentales de motivación para realizar cualquier tarea: la motivación intrínseca, en la que un comportamiento concreto se llevaría a cabo por la satisfacción y el placer inherente de la propia participación, y la motivación extrínseca en la que el motivo de participación vendría promovido por los beneficios consecuentes de dicho comportamiento separados de la propia participación. Ante estas dos formas movilizadoras, se presupone una forma de amotivación que supondría una ausencia de motivación y cierta falta de intencionalidad en la participación en una actividad concreta. A partir de estas tres formas de regulación del comportamiento, Deci y Ryan (1985) perfilan un continuo de formas motivacionales de menos a más autodeterminadas. La amotivación se consideraría como no regulada y por lo tanto como la forma menos autodeterminada de actuación. Dentro de las formas extrínsecas encontramos cuatro niveles de regulación de más externa a más internamente reguladas: la regulación externa se refiere a una conducta regulada por motivos externos. La regulación introyectada tiene que ver con la internalización de las presiones externas donde la participación se efectuaría o bien para evitar sentimientos de culpa o ansiedad o bien para lograr sentirse orgulloso de uno mismo. La regulación identificada se describe como la participación basada en la expectativa de obtención de ciertos beneficios valiosos asociados a la participación. La regulación integrada se refiere a aquellas actividades que se llevan a cabo porque se considera que constituyen parte de uno mismo. Finalmente, la motivación intrínseca, cuyo motivo sería la propia participación, representaría la forma de regulación más autodeterminada.

En posteriores revisiones, tanto desde el punto de vista teórico como desde diferentes trabajos empíricos (Deci y Ryan, 2000; Lonsdale, Hodge, y Rose, 2009; Ryan y Connell, 1989; Vallerand, 2007), se ha reestructurado el continuo de autodeterminación en función de la percepción de causalidad de la conducta, en la que se mantendría la amotivación como forma en la que la conducta se percibe como no intencionada y sin efectos sobre el entorno; la regulación controlada (que englobaría la externa y la introyectada) se percibe como una regulación de la conducta llevada a cabo por presiones y dirección externa; y la regulación autónoma (que englobaría la intrínseca, la integrada y la identificada) que se percibe como una conducta causada por intereses y valores del propio individuo. Estas formas de regulación, según el modelo que se plantea, podrían tener distintos efectos emocionales, cognitivos y conductuales sobre el individuo. Las formas más autónomas proporcionarían mayor satisfacción y bienestar, mejor rendimiento en la propia actividad y, a largo plazo, consecuencias positivas sobre la salud mental, mientras que la amotivación y la regulación controlada, implicarían menor rendimiento y satisfacción en la práctica y consecuencias de ansiedad y depresión a largo plazo.

Dado que diferentes estudios plantean el papel facilitador de los diferentes agentes de socialización en la motivación de los deportistas (Quested y Duda, 2009; 2010; Mouratidis,
Lens, y Vansteenkiste, 2010; Ntoumanis y Vazou, 2005; White, 1996), el objetivo de este trabajo ha sido analizar la relativa capacidad predictiva de la percepción de apoyo a la autonomía de entrenadores, compañeros y padres sobre la regulación motivacional de una muestra de deportistas de iniciación. En base a las investigaciones revisadas, nuestra hipótesis presume que un mayor apoyo a la autonomía por parte de los agentes de socialización, provocará formas de regulación más autónomas mientras que un menor apoyo a la autonomía, provocará formas más controladas y mayor amotivación.

\section{Método}

\section{Participantes}

Los participantes han sido 278 deportistas federados de edades comprendidas entre los 11 y los 17 años $(M=13.87$; $D T=1.68$ ) principalmente de deportes de equipo (i.e., baloncesto, fútbol, balonmano) pero incluyendo también un $10 \%$ de participantes en deportes individuales (i.e., natación, gimnasia, bádminton). La representación de chicas fue del $18 \%$ frente a una mayoría de chicos, datos que reflejan la distribución de participación por géneros en deportes federados, a nivel tanto del estado español como de Cataluña, donde se realizó el muestreo (Consejo Superior de Deportes, 2010; Consell Català de l’Esport, 2008).

\section{Instrumentos}

Apoyo a la Autonomía. La percepción de apoyo a la autonomía de los participantes se midió con la versión española del Cuestionario de Clima en el Deporte (S-SCQ; Balaguer, Castillo, Duda, y Tomás, 2009). Los 15 ítems de la escala, construida originalmente para evaluar la percepción del apoyo a la autonomía generado por los entrenadores, fueron redactados cambiando el sujeto de forma que permitieran evaluar paralelamente el apoyo a la autonomía tanto por parte de compañeros como por parte de padres. En cada versión se llevaron a cabo ligeras modificaciones de cara a recoger las funciones específicas tanto de los compañeros como de los padres y madres en el ámbito deportivo (e.g., el ítem "Mi entrenador me ofrece distintas alternativas y opciones" cuando se adaptó para compañeros se formulaba "Mis compañeros aceptan que en el equipo existen distintas alternativas y opciones"). Los deportistas valoran frases como "Me siento capaz de compartir mis sentimientos con mi entrenador (compañeros, padre o madre)" a partir de una escala Likert que va de 1 (Completamente Falso) a 7 (Completamente Verdadero). Para todos los casos una puntuación alta significa mayor percepción de apoyo a la autonomía por parte de ese agente y viceversa. Respecto del Apoyo a la Autonomía del padre y de la madre, aunque se midieron por separado, se hizo la media entre ambas siguiendo la recomendación de trabajos previos en este ámbito (Soenens, Vansteenkiste, Du- 
riez, y Goossens, 2009; Soenens, Vansteenkiste, y Sierens, 2006).

Motivación Autodeterminada. La motivación de los deportistas se evaluó mediante la versión validada en español de Viladrich, Torregrosa, y Cruz (2011) del Cuestionario de Regulación Conductual en el Deporte (BRSQ; Lonsdale, Hodge, y Rose, 2008). Esta escala de 24 ítems, se utiliza para evaluar los motivos de participación en el deporte a partir de seis subescalas que incluyen Motivación Intrínseca, Motivación Extrínseca (dividida en Externa, Introyectada, Identificada e Integrada) y Amotivación, cada una de ellas de cuatro ítems. Todos los ítems comparten la raíz "Practico este deporte..." seguida del contenido diferencial por subescala (e.g., "porque me gusta"; "porque me siento presionado por los demás para seguir haciéndolo"; "a pesar de que me pregunto por qué continúo"). Los ítems se valoran de 1 (Completamente Falso) a 7 (Completamente Verdadero). Para todas las variables una puntuación mayor significa mayor percepción de esa forma de regulación en la práctica del deporte y viceversa. Para el análisis de este cuestionario se generaron tres variables correspondientes a la Motivación Autónoma (a partir de la media obtenida en las escalas Intrínseca, Integrada e Identificada), Motivación Controlada (a partir de la Introyectada y la Externa) y Amotivación. Los datos de consistencia interna para esta muestra de las variables utilizadas en este estudio se han analizado mediante el coeficiente Alfa de Cronbach y se relatan en el apartado de resultados.

\section{Procedimiento}

Una vez los clubes y sus entrenadores accedieron a participar, se concretaron fechas y horarios para la administración de los cuestionarios. Se convocaba a los deportistas veinte minutos antes del entrenamiento y contestaban el cuadernillo o bien en el vestuario o bien en aulas facilitadas por los clubes. De cara a evitar sesgos por género, los cuestionarios se construyeron en dos versiones con los ítems redactados en femenino y en masculino. El protocolo de aplicación establecía que como mínimo dos investigadores debían estar presentes en todo momento de cara a resolver las posibles dudas de los deportistas. Se informó previamente a los deportistas del motivo del estudio y de la duración de la administración, así como de la confidencialidad de los datos obtenidos y todos mostraron su conformidad de cara a participar en la investigación. Una vez respondidos los cuestionarios, procedieron a su rutina habitual de entrenamiento.

Para el tratamiento de los datos faltantes se optó por una imputación simple basada en la sustitución de éstos por la puntuación media de la persona en esa subescala. Al ser el número de valores perdidos menor al 5\% del total de puntos de datos, esta imputación no tiene consecuencias sobre los análisis realizados (Graham, 2009). No obstante, debido al tipo de imputación, fueron excluidos aquellos participantes que habían dejado sin contestar una o varias de las escalas del cuadernillo de cuestionarios, por lo que la muestra final analizada fue de 269 participantes.

\section{Resultados}

Estadísticos descriptivos y análisis de fiabilidad. En la Tabla 1 se presentan los estadísticos descriptivos (media y desviación estándar) de las diferentes escalas que se analizaron mediante el paquete estadístico SPSS 17.0. Observamos que respecto al apoyo a la autonomía se obtienen valores medios altos tanto respecto de entrenadores como de compañeros y padres, siendo las puntuaciones mayores las generadas por los padres, seguidas por las de compañeros y entrenadores. Lo mismo pasa respecto de la regulación autónoma, muy por encima de la regulación controlada con un valor que se acerca a los puntos mínimos del rango potencial de la escala de respuesta y que es incluso menor para el factor de amotivación.

En lo que a fiabilidad de las escalas se refiere, en la Tabla 1 observamos que la consistencia interna de la mayoría de ellas es aceptable, superando para las tres medidas de apoyo a la autonomía el valor 90 y siendo la amotivación la única de todas que no llega al criterio de .70 establecido por Nunnally (1978) para la investigación en el ámbito de la psicología, aunque con un valor muy cercano a él (.68).

Tabla 1. Estadísticos descriptivos para las diferentes escalas de apoyo a la autonomía y regulación conductual

\begin{tabular}{lllll}
\hline & Media & DT. & N $^{\circ}$ de ítems & Alfa \\
\hline A.A. Entrenador & 5.05 & 1.32 & 15 & .93 \\
A.A. Compañeros & 5.39 & 1.07 & 15 & .91 \\
A.A. Padres & 5.59 & 1.04 & 15 & .93 \\
Regulación Autónoma & 5.64 & 0.90 & 12 & .82 \\
Regulación Controlada & 2.01 & 1.07 & 8 & .78 \\
Amotivación & 1.90 & 1.15 & 4 & .68 \\
\hline
\end{tabular}

Nota: Todos los ítems tienen rango de 1 a 7 ;

“A.A." - Apoyo a la Autonomía; DT - Desviación típica

Análisis del Modelo Estructural. Se realizó un análisis del modelo estructural mediante el programa de modelado estadístico Mplus 6.0 (Muthén y Muthén, 1998-2010). Se definieron tres variables independientes que correspondían al Apoyo a la Autonomía de entrenadores, compañeros y padres, y tres variables dependientes que correspondían a la regulación autónoma, la regulación controlada y la amotivación de los deportistas, permitiendo que tanto las independientes como las dependientes correlacionaran entre sí (ver Figura 1). Se obtuvieron correlaciones positivas y significativas para el apoyo a la autonomía entre los tres agentes de socialización, siendo la más alta la que se da entre los agentes dentro del propio equipo deportivo: entrenadores y compañeros. Para las variables dependientes, fueron significativas y positivas las correlaciones entre la regulación autónoma y la controlada, y entre la controlada y la amotivación. La correlación obtenida entre la regulación autónoma y la amotivación no fue estadísticamente significativa.

En referencia a la influencia del apoyo a la autonomía sobre las formas de regulación conductual de los deportistas, se observa que el apoyo generado por los entrenadores, padres y compañeros predice en positivo la regulación autó- 
noma de los deportistas. El elemento principal es el apoyo a la autonomía por parte de los entrenadores $(\beta=.25)$, seguido del de los padres y el de los compañeros $(\beta=.16$ y $\beta=.11$, respectivamente), todos ellos efectos estadísticamente significativos. Concretamente, la percepción de que los entrenadores, los padres y los compañeros apoyen la autonomía de los deportistas, influye positivamente en el desarrollo de una regulación más autónoma por parte de estos. Respecto de la amotivación, los resultados muestran que nuevamente esta viene predicha por el apoyo a la autonomía del entrenador, aunque la influencia es negativa en este caso $(\beta=-.26)$. Es decir que el entrenador promueva un estilo de apoyo a la autonomía de sus jugadores hace que los deportistas manifiesten significativamente menos amotivación sobre la práctica de su deporte. No se encontraron influencias significativas sobre la regulación controlada por parte de ninguno de los agentes estudiados.

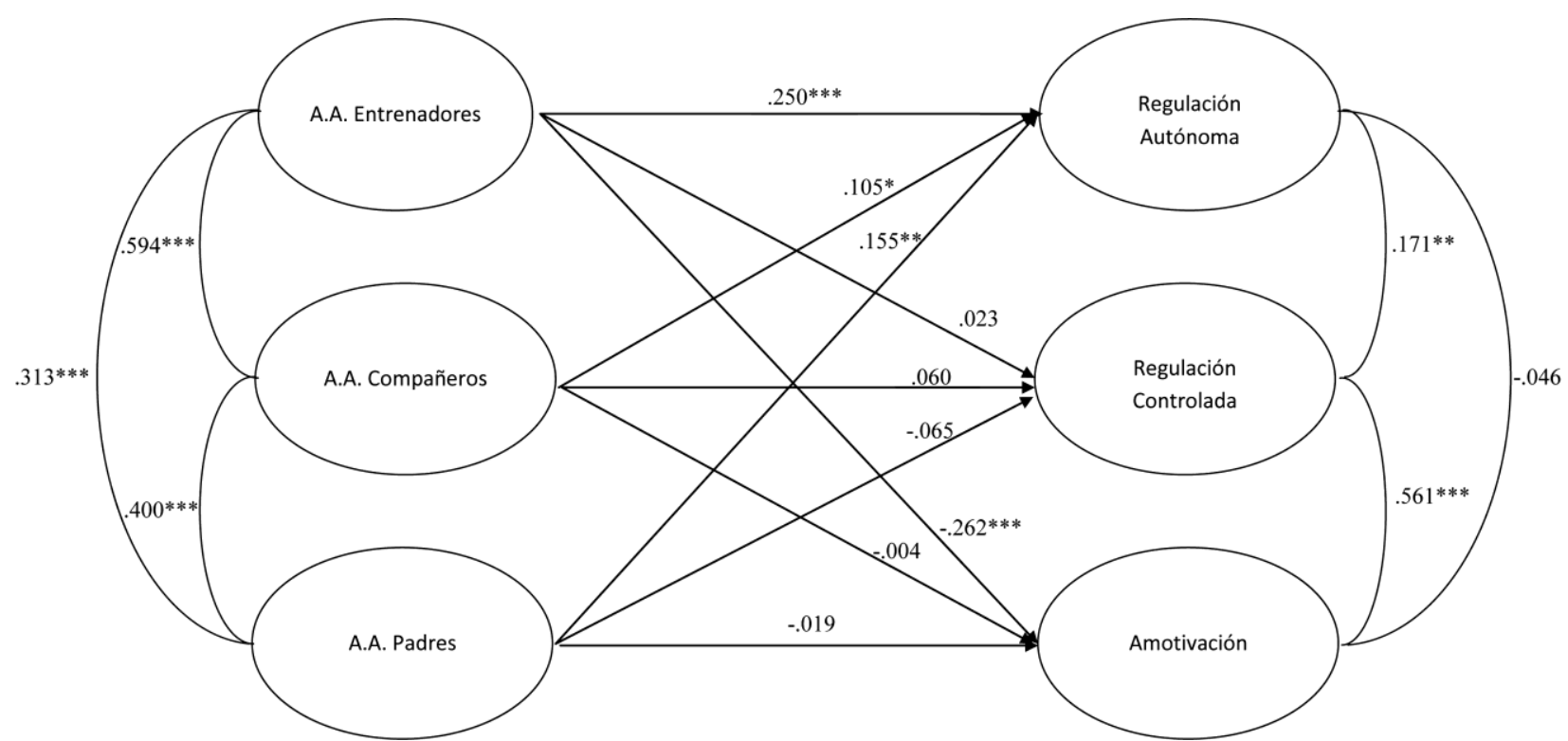

Figura 1. Modelo estructural de la regresión entre el apoyo a la autonomía sobre la regulación conductual de los deportistas. Nota: "A.A."-Apoyo a la Autonomía; ${ }^{*} p<.05 ;{ }^{* *} p<.01 ;{ }^{* *} p<.001$

\section{Discusión}

Este trabajo aporta nuevas evidencias acerca de la importancia del entorno y el apoyo a la autonomía de los agentes sociales que lo conforman respecto de la motivación de los participantes en el ámbito deportivo. Los resultados obtenidos en los análisis del modelo estructural muestran que el apoyo a la autonomía tanto de entrenadores como de padres y compañeros predice la regulación autónoma. No obstante, el grado de influencia de cada uno de los agentes varía ostensiblemente. El entrenador se destaca como el elemento clave siendo su influencia notablemente mayor que la de los compañeros y mayor incluso que la generada por los padres. Estos resultados irían en la línea de lo que propone Vallerand (2007), donde el papel de los diferentes agentes del entorno del individuo tendría influencias distintas en función del contexto específico en que se produzca, y si bien la familia podría tener efectos a nivel global puesto que influye en la mayoría de contextos de los niños y niñas (Assor, et al., 2004), en el contexto deportivo el elemento fundamental de referencia sería el entrenador.

En la misma línea, hemos comprobado que el elemento que predice mejor la amotivación de los deportistas de la muestra estudiada, aunque en negativo en este caso, es preci- samente el apoyo a la autonomía de los entrenadores, siendo este el único agente de socialización que presenta una relación significativa con la forma no regulada de motivación. Dado que la amotivación se relacionaría con la percepción del individuo de que su actividad no tiene efecto sobre el entorno en que se produce (Deci y Ryan, 2000), es explicable que la conducta de apoyo a la autonomía por parte del entrenador sea la variable que mejor proteja de la aparición de amotivación en sus deportistas puesto que es precisamente el entrenador el agente más influyente en la definición del contexto deportivo. Este resultado significa que la percepción de las chicas y chicos de que sus entrenadores los dotan de poder de decisión haciéndolos partícipes de lo que sucede dentro del equipo, refuerza los motivos de participación protegiendo así de la no regulación.

Respecto de la regulación controlada no se han observado predicciones significativas desde el apoyo a la autonomía de los agentes estudiados. Si bien esto va en contra de la hipótesis que planteábamos inicialmente en la que el apoyo a la autonomía predeciría negativamente las formas de regulación controlada, la literatura reciente plantea que así como el apoyo a la autonomía es un buen predictor de las consecuencias motivacionales positivas (e.g., regulación autónoma), son las medidas que evalúan la rigidez o el control del entorno las 
que tienen mejor capacidad predictiva sobre las negativas (Bartholomew, Ntoumanis y Thogersen-Ntoumani, 2010; Pelletier, Fortier, Vallerand y Brière, 2001). Este enfoque, más allá de una justificación psicométrica, propondría un planteamiento teórico bajo el cual el apoyo a la autonomía y el estilo controlador no serían extremos de un mismo continuo, sino variables independientes entre sí. La ausencia de apoyo a la autonomía en la percepción de un agente social podría indicar sencillamente un estilo neutral sin implicar directamente mayor control sobre el individuo.

Aunque no se había planteado como objetivo principal de nuestro trabajo, el análisis de correlaciones plantea una serie de resultados interesantes. Inicialmente, destaca que las percepciones de apoyo a la autonomía de los tres agentes estudiados correlacionan positiva y significativamente entre ellas. En cuanto a magnitudes, la correlación más alta es la que se produce entre entrenadores y compañeros. Si asociamos nuevamente este resultado a la conceptualización del modelo jerárquico de Vallerand (2007), en la que un contexto específico viene definido por los agentes sociales significativos que lo conforman, o con otros estudios que evalúan precisamente la relación entre el clima generado por entrenadores y compañeros (Vazou, 2010), podemos explicar que en un mismo equipo deportivo, entrenadores e integrantes del equipo sean percibidos de manera similar. Por su parte, en cuanto a las formas de regulación, comprobamos que la regulación autónoma correlaciona de forma positiva con la controlada aunque con una magnitud muy discreta y que la controlada correlaciona positivamente con la amotivación siendo la magnitud de estas mucho mayor. Estos resultados apoyan la conceptualización progresiva de la autodeterminación (Deci y Ryan, 2000) en la que la no regulación se encontraría en un extremo y la motivación más autodeterminada en el otro, actuando las formas extrínsecas de puente entre ellas. Si bien en nuestro estudio el uso de las variables agrupadas Regulación Autónoma y Regulación Controlada puede haber diluido este efecto, estudios recientes sobre la regulación conductual han revisado y reafirman esta conceptualización progresiva (Viladrich et al, 2011).

Algunas de las limitaciones que cabría recalcar del trabajo presentado vienen desde el punto de vista del modelo teórico en el que nos hemos enmarcado, puesto que el modelo jerárquico de Vallerand (2007) propone que, al margen de la influencia directa de los agentes de socialización sobre el tipo de regulación, existe el factor mediador de las necesidades básicas de autonomía, competencia y relación que en este estudio no han sido evaluadas y que en el futuro, sería interesante tomar en consideración. Para completar la red nomológica de la autodeterminación, se deberían también estudiar los consecuentes que una u otra regulación puede tener sobre variables afectivas (e.g., ansiedad competitiva; Ramis, Torregrosa, Viladrich y Cruz, 2010), cognitivas (e.g., contenido de pensamiento; Latinjak, Torregrosa y Renom, 2011) y conductuales (e.g., deportividad; Borràs, Palou, Ponseti, Vidal y García-Mas, 2009) de los participantes del deporte de iniciación a partir de instrumentos validados y adaptados. Además, aunque en nuestro trabajo no hemos controlado la variable género debido a la reducida representación femenina, estudios recientes han demostrado que podrían existir diferencias en como los chicos y chicas perciben a sus entrenadores y compañeros de equipo (Torregrosa et al., 2011) por lo que recomendamos que esta variable sea tomada en consideración en futuros trabajos.

Nuestro trabajo ha abordado simultáneamente la percepción del apoyo a la autonomía de los entrenadores, padres y compañeros de equipo, para evaluar la varianza explicada por cada uno de ellos, respecto de la regulación conductual de los deportistas. Si bien el estudio ha sido planteado desde la perspectiva de la teoría de la autodeterminación, aborda una temática ya revisada desde otros modelos que llegaron a conclusiones similares en las que el entrenador se postulaba como el elemento clave de socialización una vez que los deportistas entraban en el deporte organizado. Desde la perspectiva de las metas de logro en el trabajo con deportistas de iniciación (Boixadós, Cruz, Torregrosa, y Valiente, 2004; Duda, 2001) o desde el enfoque de las transiciones de carrera con deportistas de alto rendimiento (Torregrosa, Sánchez, y Cruz, 2004; Wylleman y Lavallee, 2004), se recalca la importancia del entrenador para promover tanto la participación, el compromiso y la motivación de los deportistas, como las consecuencias que conllevan tanto positivas (e.g. flujo disposicional; Moreno, Cervelló, y González-Cutre, 2006, 2010) como las negativas (e.g., estrés y burnout; Julien, Guay, Senécal, y Poitras, 2009; Lonsdale et al., 2009). Por esta razón, trabajos de enfoque teórico, como es nuestro caso, deberían ser complementados con investigaciones de intervención en los que se promoviera el apoyo a la autonomía de los diferentes agentes sociales para favorecer la máxima autodeterminación de los deportistas jóvenes fomentando el llamado "perfil autodeterminado de motivación" (Sicilia, Águila, Muyor, Orta y Moreno, 2009) y garantizando así su continuidad en el deporte organizado o como mínimo el compromiso con el hábito de la actividad física saludable.

Asumiendo que la motivación es uno de los motores de la conducta humana y manteniendo como cierto que la motivación de un ámbito específico puede afectar a la motivación general y a la satisfacción de las necesidades básicas del individuo, es importante no dejar de lado un ámbito de socialización como es el ámbito deportivo.

Agradecimientos.- Este trabajo se ha realizado en parte gracias a las subvenciones del Ministerio de Ciencia e Innovación DEP2010-15561 y de la Unión Europea F2-223600. Los autores quieren agradecer a Saül Alcaraz, Jesús Portillo y Verónica Levineri la colaboración en la recogida y gestión de los datos. 


\section{Referencias}

Assor, A., Roth, G., y Deci, E. L., (2004). The emotional costs of parents' conditional regard: A self-determination theory analysis. Journal of Personality, 72, $47-88$.

Balaguer, I., Castillo, I., Duda, J. L., y Tomás, I. (2009). Análisis de las propiedades psicométricas de la versión española del cuestionario de clima en el deporte. Revista de Psicologia del Deporte, 18, 73-83.

Bartholomew, K.J., Ntoumanis, N., y Thogersen-Ntoumani, C. (2010). The controlling interpersonal style in a coaching context: Development and inicial validation of a psychometric scale. Journal of Sport and Exercise Psychology, 32, 193-216.

Black, A. E., y Deci, E. L. (2000). The effects of instructors' autonomy support and students' autonomous motivation on learning organic chemistry: A selfdetermination theory perspective. Science Education, 84, 740-756.

Boixadós, M., Cruz, J., Torregrosa, M., y Valiente, L. (2004). Relationships among motivational climate, satisfaction, perceived ability, and fair play attitudes in young soccer players. Journal of Applied Sport Psychology, 16, 301-317.

Borràs, P.A.; Palou, P.; Ponseti, F.J.; Vidal, J.; Garcia-Mas, A.; (2009). La educación en valores en la práctica deportiva de los adolescentes: efectos de una intervención para la promoción de la deportividad sobre la estructura de valores de los deportistas. Revista Española de Pedagogia, 243, 355-370.

Brustad, R. J. (1996). Parental and peer influence on children's psychological development through sport: En F.L. Smoll y R.C. Smith (Eds.). Children and youth in sport: a byopsychosocial perspective. (pp. 112-124). Dubuque, IA: Brown \& Benchmark

Consell Català de l'Esport (2008). Pràctica esportiva en edat escolar. Recuperado el 18 de Junio del 2010 de

http://www.observatoridelesport.cat/cat/subdimensio.asp?ID_SUBDIME $\mathrm{NSIO}=4 \&$ ID_DIMENSIO $=1$

Consejo Superior de Deportes (2012). Los hábitos deportivos de la población escolar en España. Recuperado el 21 de Enero de 2012 de http://www.infocoponline.es/pdf/Estudio\%20hábitos\%20deportivos.pdf

Deci, E. L. (1975). Intrinsic motivation. New York: Plenum.

Deci, E. L., y Ryan, R. M. (1985). Intrinsic motivation and self-determination in buman behavior. New York: Plenum.

Deci, E. L., y Ryan, R. M. (1987). The support of autonomy and the control of behavior. Journal of Personality and Social Psychology, 53, 1024-1037.

Deci, E. L., y Ryan, R. M. (2000). The "what" and "why" of goal pursuits: Human needs and the self-determination of behaviour. Psychological Inquiry, 11(4), 227-268.

Duda, J.L. (2001) Achievement goal research in sport: Pushing the boundaries and clarifying some misunderstandings. En G.C. Roberts (Ed.), Advances in motivation in sport and exercise (pp. 129-182). Champaign, IL: Human Kinetics.

Graham, J. W. (2009). Missing data analysis: making it work in the real world. Annual Review of Psychology, 60, 549 - 576.

Hagger, M.S., y Chatzisarantis, N.L.D. (2005). The social psychology of exercise and sport. Buckingham, UK: Open University Press.

Julien, E., Guay, F., Sénecal, C., y Poitras, S.C. (2009). Subjective psychological distress among young adults: The role of global and contextual levels of selfdetermined motivation. Hellenic Journal of Psychology, 6, 145-168.

Latinjak, A., Torregrosa, M., y Renom, J. (2011). Studying the effects of self talk on thought contents with male adult tennis players. Perceptual and Motor Skills, 111(1), 249-260.

Lonsdale, C., Hodge, K., y Rose, E.A. (2008). The Behavioral Regulation in Sport Questionnaire (BRSQ): Instrument development and initial validity evidence. Journal of Sport and Exercise Psychology, 30, 323-355.

Lonsdale, C., Hodge, K., y Rose, E.A. (2009).Athlete burnout in elite sport: A self-determination perspective. Journal of Sports Sciences, 27, 785 - 795.

Moreno, J.A., Cervelló, E., y González-Cutre, D. (2006). Motivación autodeterminada y flujo disposicional en el deporte. Anales de Psicología, 22, 310-317.

Moreno, J.A., Cervelló, E., y González-Cutre, D. (2010). The achievement goal and self-determination theories as predictors of dispositional flow in young athletes. Anales de Psicología, 26, 390-399.

Mouratidis, A., Lens, W., y Vansteenkiste, M. (2010). How you provide corrective feedback makes a difference: The motivating role of communicating in an autonomy-supporting way. Journal of Sport and Exercise Psychology, 32, 619 637.

Muthén, L.K., y Muthén, B.O. (1998-2010). MPlus versión 6.0 [programa de ordenador]. Los Angeles, CA: Muthén \& Muthén.

Ntoumanis, N., y Vazou, S., (2005). Peer motivational climate in youth sport: Measurement development and validation. Journal of Sport and Exercise Psychology, 27, 432-455.

Nunnally, J.C. (1978). Psychometric theory (2nd edition). New York: McGraw-Hill.

PASW Statistics (versión 17) [Programario informático]. Chicago, IL: SPSS Inc.

Pelletier, L.G., Portier, M.S., Vallerand, R.J., y Brière, N.M. (2001). Associations among perceived autonomy support, forms of self-regulation, and persistence: A prospective study. Motivation and Emotion, 25, 279-306.

Quested, E., y Duda, J.L. (2009). Perceptions of the motivational climate, need satisfaction, and indices of well- and ill-being among hip hop dancers. Jour nal of Dance Medicine and Science, 13, $10-19$.

Quested, E., y Duda, J.L. (2010). Exploring the social environmental determinants of well- and ill-being in dancers: A test of basic needs theory. Journal of Sport and Exercise Psychology, 32, 39 - 60.

Ramis, Y., Torregrosa, M, Viladrich, C. y Cruz, J. (2010). Adaptación y validación de la versión española de la Escala de Ansiedad Competitiva SAS-2 para deportistas de iniciación. Psicothema, 22, 1004-1009.

Ryan, R. M., y Connell, J. P. (1989). Perceived locus of causality and internalization: Examining reasons for acting in two domains. Journal of Personality and Social Psychology, 57, 749-761

Sicilia, Á., Águila, C., Muyor, J.M., Orta, A., y Moreno, J.A. (2009). Perfiles motivacionales de los usuarios en centros deportivos municipales. Anales de Psicologia, 25, 160-168.

Smith, A. (2003). Peer relationships in physical activity contexts: a road less travelled in youth sport and excercise psychology research. Psychology of Sport and Excercise, 4, 25-39.

Soenens, B., Vansteenkiste, M., Duriez, B., y Goossens, L. (2006). In search of psychologically controlling parenting: The role of parental separation anxiety and parental maladaptive perfectionism. Journal of Research on Adolescence, 16, $539-559$.

Soenens, B., Vansteenkiste, M., y Sierens, E. (2009). How are parental psychological control and autonomy support related? A cluster-analytic approach. Journal of Marriage, 71, 187 - 202

Torregrosa, M., Sánchez, X., y Cruz, J. (2004). El papel del psicólogo del deporte en el asesoramiento académico-vocacional del deportista de elite. Revista de Psicología del Deporte, 13, 215-288.

Torregrosa, M., Viladrich, C., Ramis, Y., Azócar, F., Latinjak, A.T., y Cruz, J. (2011). Efectos en la percepción del clima motivacional generado por los entrenadores y compañeros sobre la diversión y el compromiso. Diferencias en función del género. Revista de Psicología del Deporte, 20, 243-255.

Vallerand, R.J. (2007). A hierarchical model of intrinsic and extrinsic motivation for sport and physical activity. En M.S. Hagger, y L.D. Chatzisarantis (Eds.), Intrinsic motivation and self-determination in exercise and sport (pp. 255-279). Champaign, IL: Human Kinetics.

Vallerand, R. J., Fortier, M. S., y Guay, F. (1997). Self-determination and persistence in a real-life setting: Toward a motivational model of high school dropout. Journal of Personality and Social Psychology, 72, 1161-1176.

Vazou, S., (2010). Variations in the perceptions of peer and coach motivational climate. Research Quarterly for Exercise and Sport, 81, 199-211.

Viladrich, C., Torregrosa, M., y Cruz, J. (2011). Calidad Psicométrica de la adaptación española del Cuestionario de Regulación Conductual en el Deporte. Psicothema, 23, 786-794.

White, S.A., (1996). Goal orientation and perceptions of the motivational climate initiated by parents. Pediatric Exercise Science, 8, 122-129.

Wylleman, P., y Lavallee, D. (2004). A developmental perspective on transitions faced by athletes. En: M. Weiss (Ed.), Developmental sport and exercise psychology: a lifespan perspective (pp. 507-527). Morgantown, WV: FIT

(Artículo recibido: 29-03-2011, revisión: 31-01-2012, aceptado: 01-02-2012) 